\title{
Pituitary Gland Functional Connectivity and BMI
}

\author{
Paige Rucker ${ }^{1}$ and Toshikazu lkuta ${ }^{2 *}$ \\ ${ }^{1}$ School of Medicine, The University of Mississippi Medical Center, Jackson, MS, United States, ${ }^{2}$ Department of \\ Communication Sciences and Disorders, The University of Mississippi, Oxford, MS, United States
}

The pituitary gland (PG) influences body weight through hormonal releases; however, the relation between body weight and PG's co-activities with other brain regions remains unclear. Here, we aimed to identify (1) the functional connectivity of the PG and (2) $P G$ functional connectivity associated with body mass index by examining resting state functional magnetic resonance imaging data. Using enhanced Nathan Kline InstituteRockland Sample, PG functional connectivity of 494 individuals was analyzed to assess in voxel-wise fashion. A negative association was found between BMI and PG functional connectivity with the orbitofrontal cortex, hippocampus, putamen, and temporal lobe. Our results show PG dysconnectivity to these regions is associated with higher BMl and implicate that the connectivity between these dopaminergic regions and PG may be associated with body weight maintenance through feeding behavior and growth.

\section{OPEN ACCESS}

Edited by: Joana M. Gaspar. Universidade Federal de Santa Catarina, Brazil

Reviewed by: João M. N. Duarte, Lund University, Sweden Ana Lucía De Paul, Universidad Nacional de Córdoba, Argentina

*Correspondence: Toshikazu lkuta tikuta@olemiss.edu

Specialty section: This article was submitted to Neuroenergetics, Nutrition and Brain

Health,

a section of the journal

Frontiers in Neuroscience

Received: 07 August 2018

Accepted: 01 February 2019

Published: 01 March 2019

Citation:

Rucker P and Ikuta T (2019)

Pituitary Gland Functional

Connectivity and BMI.

Front. Neurosci. 13:120.

doi: 10.3389/fnins.2019.00120
Keywords: pituitary gland, functional connectivity, resting state fMRI, gustatory cortex, caudate head

\section{INTRODUCTION}

The hypothalamic-pituitary-adrenal (HPA) axis including the pituitary gland (PG) has been shown to be associated with obesity (Pasquali et al., 2006). In obesity, responses to growth hormone releasing factor are impaired (Williams et al., 1984). Chronic stress has been implicated to promote obesity through the HPA axis (Bose et al., 2009). Genetic variation in leptin receptors also shows association with obesity (Clement et al., 1998). The PG synthesizes and secretes hormones that regulate body weight, including somatotropins and oxytocin; however, the brain regions responsible for associating the PG activity and obesity are not clearly known.

Functional connectivity analysis using resting state fMRI (Fox and Raichle, 2007; Biswal et al., 2010) has provided newer insights into brain coactivity and obesity. The default mode network (DMN) and temporal lobe network have been found to be associated with obesity (Kullmann et al., 2012). In obesity, the precuneus shows an increased functional connectivity in the DMN while that of the anterior cingulate cortex is decreased. The insular cortex shows reduced connectivity in the temporal lobe network. Dysconnectivity between the hypothalamus and left insula has been found in obesity (Wijngaarden et al., 2015). After $48 \mathrm{~h}$ of fasting, connectivity of the hypothalamus to the dorsal anterior cingulate cortex increases in lean populations and decreases in the obese populations, suggesting a differential influence of obesity to functional connectivity of the hypothalamus (Wijngaarden et al., 2015). The putamen has been found to have elevated functional connectivity in obesity, while cognitive processing speed was negatively associated with the connectivity of the putamen to the salience network, suggesting an altered processing of salience detection (García-García et al., 2013). Obesity-preventive eating tendency has been found to be associated with caudate-precuneus functional connectivity (Nakamura and Ikuta, 2017). Caudate-precuneus connectivity inversely predicts the personal characteristics of avoiding 
obesity-inducing behaviors, suggesting the functional connectivity signature of avoiding causes of obesity independent of current body weight status. Surgical and behavioral weight loss has been found to differentially influence functional connectivity (Lepping et al., 2015). Despite its strong relationships to obesity, the influence of functional connectivity of the PG to body weight has not been yet studied.

Resting state functional connectivity of the PG is minimally studied, although functional connectivity is associated with adrenocorticotropic hormone (ACTH) levels. Cortisol concentrations have been found to predict interhemispheric connectivity, and ACTH concentrations were shown to be associated with the subcallosal anterior cingulate cortex (Kiem et al., 2013). Nonetheless, the resting state PG connectivity remains unclear. In this study, we (1) examined the resting state functional connectivity of the PG and (2) aimed to isolate resting state functional connectivity of the PG associated with BMI.

\section{MATERIALS AND METHODS}

\section{Data Acquisition}

The MRI images, the clinical data, and the demographic data of the enhanced Nathan Kline Institute-Rockland Sample (Nooner et al., 2012) were obtained from Collaborative Informatics and Neuroimaging Suite (Biswal et al., 2010). This data subset consisted of 494 individuals without known neurological preconditions (such as stroke, tumor, and traumatic brain damage) and MRI counter indications (43.46 \pm 20.81 years old), 310 females and 184 males, six Native Americans, 25 Asians, 102 Black or African Americans, two Native Hawaiians, 346 Caucasians, and 13 other races, with the mean BMI of $27.32 \pm 6.35$ (between 15.29 and 56.28), for whom resting state and structural data were both available. Participants in the sample were recruited from Rockland County, NY, whose demographics represent the United States (Nooner et al., 2012). The subjects with known pituitary conditions (such as pituitary tumor) were not included in the analysis data.

Resting state echo planar image (EPI) volumes had 64 slices of $2 \mathrm{~mm} 112 \times 112$ matrix with $2 \mathrm{~mm}$ thickness (voxel size $=2 \mathrm{~mm} \times 2 \mathrm{~mm} \times 2 \mathrm{~mm}), \mathrm{FOV}=224 \mathrm{~mm}$, with repetition time (TR) of $1400 \mathrm{~ms}$ and echo time (TE) of $30 \mathrm{~ms}$. A total of 404 volumes $(\sim 10 \mathrm{~min})$ were used in the analysis. Highresolution structural $\mathrm{T} 1$ volume was acquired as 176 sagittal slices of with $1 \mathrm{~mm}$ thickness (voxel size $=1 \mathrm{~mm} \times 1 \mathrm{~mm} \times 1 \mathrm{~mm}$, $\mathrm{TR}=1900 \mathrm{~ms}$ and $\mathrm{TE}=2.52 \mathrm{~ms}, \mathrm{FOV}=256)$.

\section{Data Processing}

Data processing followed previous publication (Kiparizoska and Ikuta, 2017). Data preprocessing and statistical analyses were conducted using FMRIB Software Library (FSL,) as well as Analysis of Functional NeuroImages (AFNI). The anatomical volume for each subject was skull stripped, segmented (gray matter, white matter, and CSF), and registered to the MNI $2 \mathrm{~mm}$ standard brain. First four EPI volumes were removed. Transient signal spikes were removed by de-spiking interpolation. To correct head motion, the volumes were linearly registered to the then first volume, through which six motion parameters and displacement distance between two consecutive volumes were estimated. The first volume is registered to the standard MNI152 $2 \mathrm{~mm}$ brain. Through this registration, 12 affine parameters were created between rs-fMRI volume and MNI152 $2 \mathrm{~mm}$ space, so that the processed EPI volume can later be registered to the MNI space. Each of the resting state volumes was regressed by white matter and cerebrospinal fluid signal fluctuations as well as the six motion parameters. After smoothing with a $6 \mathrm{~mm}$ FWHM Gaussian kernel, the volumes were resampled, spatially transformed and aligned to the MNI $2 \mathrm{~mm}$ standard brain space. To perform scrubbing where the volumes with excess motion are removed, as a displacement distance between two EPI volumes, the root mean square deviation was calculated from motion correction parameters, at an $r=40 \mathrm{~mm}$ spherical surface using FSL's rmsdiff tool (Power et al., 2012, 2015). Volumes whose displacement distance exceeded the threshold $(0.3 \mathrm{~mm})$ were removed (scrubbed) from further statistical analyses (Siegel et al., 2014).

The PG ROI was manually defined in the MNI $2 \mathrm{~mm}$ space centered approximately at [MNI: 0, 2, -32] (Figure 2: Red), following previous MRI literature (Klomp et al., 2012). Data were excluded if the PG was located outside of the acquisition. Voxelwise connectivity analysis was conducted in each individual brain. The time course was spatially averaged within the PG ROI that was registered to the EPI space so that correlations could be tested between the ROI and each individual voxel across the brain. The $Z$-scores representing the correlations between the ROI and a voxel were used for group level analysis after registration to the MNI $2 \mathrm{~mm}$ brain space.

In order to elucidate the regions which showed functional connectivity and dysconnectivity, one-sample $t$ test was conducted assessing correlation (positive connectivity) and anticorrelation (negative connectivity) to the PG. Using randomize script in FSL, contrast images were estimated with cluster threshold of $Z>3.72$. The association between BMI and whole brain functional connectivity to the PG was tested in a voxel-wise fashion using randomize script in FSL, taking age as a covariate. Contrast images were estimated with voxel-wise threshold of $p<0.05$ (family wise error corrected), and minimum cluster size of 10 voxels.

\section{RESULTS}

In one-sample $t$ test, in addition to the hypothalamus, the ventral and medial prefrontal cortex, inferior temporal gyrus, postcentral gyrus, insular cortex, parahippocampal gyrus, putamen, caudate head, and midbrain (periaqueductal gray), bilaterally showed positive connectivity with the PG (yellow/red in Figure 1). The dorsolateral prefrontal, parietal, occipital, and anterior cingulate cortices, hippocampus, caudate body, thalamus, pons medulla, and cerebellum showed bilateral negative connectivity (blue in Figure 1).

No regions showed positive association between the PG functional connectivity and BMI. The left orbitofrontal cortex, 


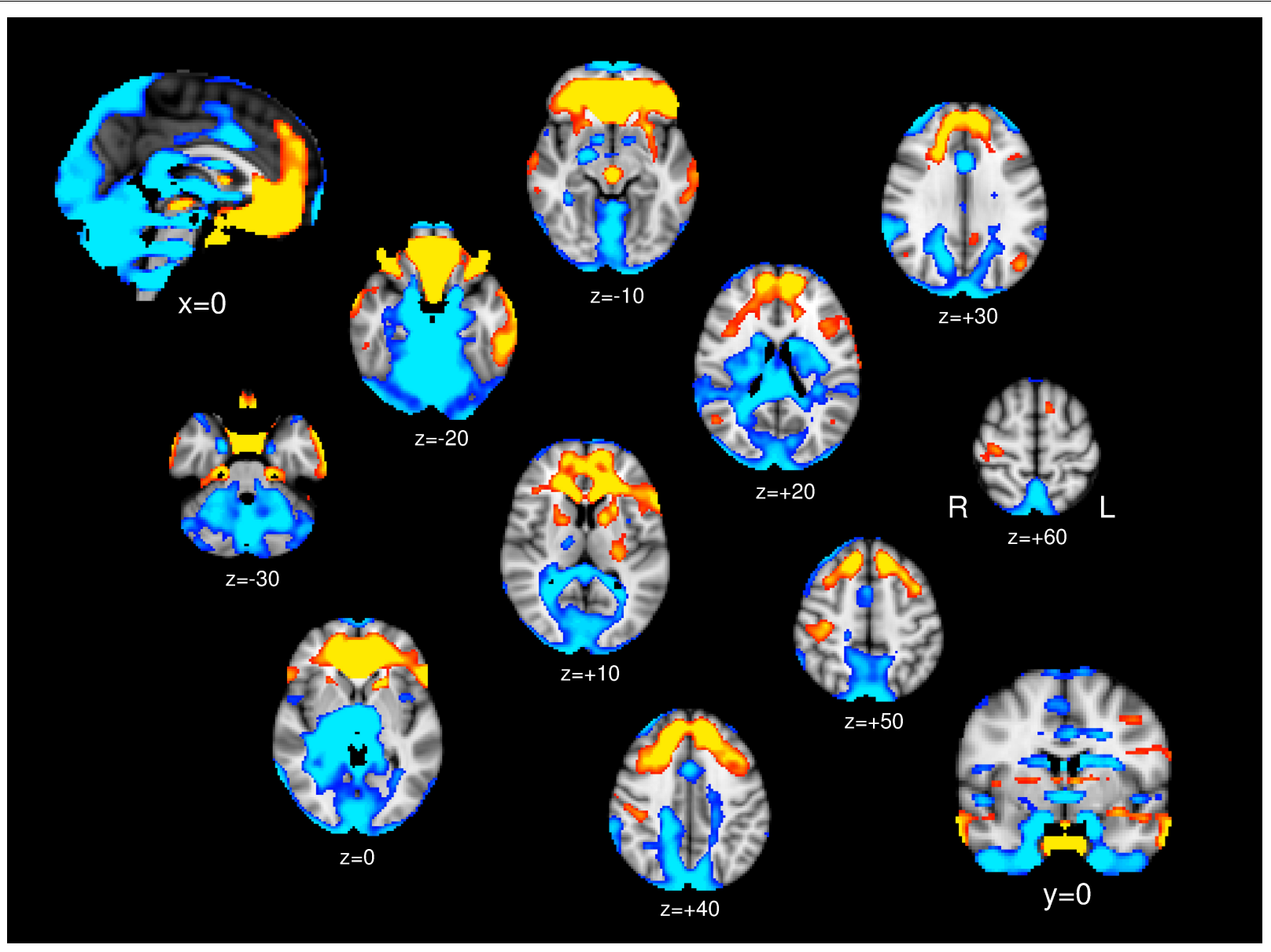

FIGURE 1 | One-sample $t$ test; Brain regions show significant positive connectivity (yellow/red) and negative connectivity (blue) to the PG.

TABLE 1 | Regions whose PG connectivity showed negative association with BMI.

\begin{tabular}{|c|c|c|c|c|c|c|c|}
\hline & \multirow[t]{2}{*}{ Voxels } & \multirow[t]{2}{*}{ Peak p (corrected) } & \multirow[t]{2}{*}{ Cluster $p$ (corrected) } & \multicolumn{3}{|c|}{ MNI coordinates } & \multirow[t]{2}{*}{ Cluster Region } \\
\hline & & & & $\mathbf{x}$ & $\mathbf{y}$ & $\mathbf{z}$ & \\
\hline 1 & 45 & 0.0002 & 0.015 & -26 & 12 & -20 & Left Orbitofrontal Cortex \\
\hline 2 & 41 & 0.0012 & 0.019 & -24 & -12 & -20 & Left Hippocampus \\
\hline 3 & 33 & 0.0064 & 0.046 & 40 & -26 & -4 & Right Superior Temporal Gyrus \\
\hline 4 & 23 & 0.0024 & 0.019 & 16 & 10 & -4 & Right Putamen \\
\hline 5 & 22 & 0.006 & 0.022 & 26 & -2 & -4 & Right Putamen/Pallidum \\
\hline 6 & 21 & 0.013 & 0.030 & -44 & 16 & -30 & Left Temporal Pole \\
\hline 7 & 14 & 0.009 & 0.024 & 16 & -36 & 0 & Right Hippocampus \\
\hline 8 & 10 & 0.023 & 0.035 & -14 & 8 & -10 & Left Putamen \\
\hline
\end{tabular}

bilateral hippocampus, bilateral putamen, and right superior temporal gyrus showed functional connectivity with the PG inversely associated with BMI (Table 1 and Figure 2).

\section{DISCUSSION}

This study aimed to identify (1) the functional connectivity of the PG and (2) brain regions whose functional connectivity to the PG shows associations with Body Mass Index. In one-sample $T$-test, the hypothalamus showed positive associations with the PG. This conforms to their close relationship classically know as a part of the HPA axis, which is known to be associated with obesity (Chalew et al., 1995). In this current study, however, the hypothalamus-PG functional connectivity did not show significant association with BMI.

Given their close relationship, we conducted an ROI-ROI connectivity analysis between the hypothalamus and PG. To 


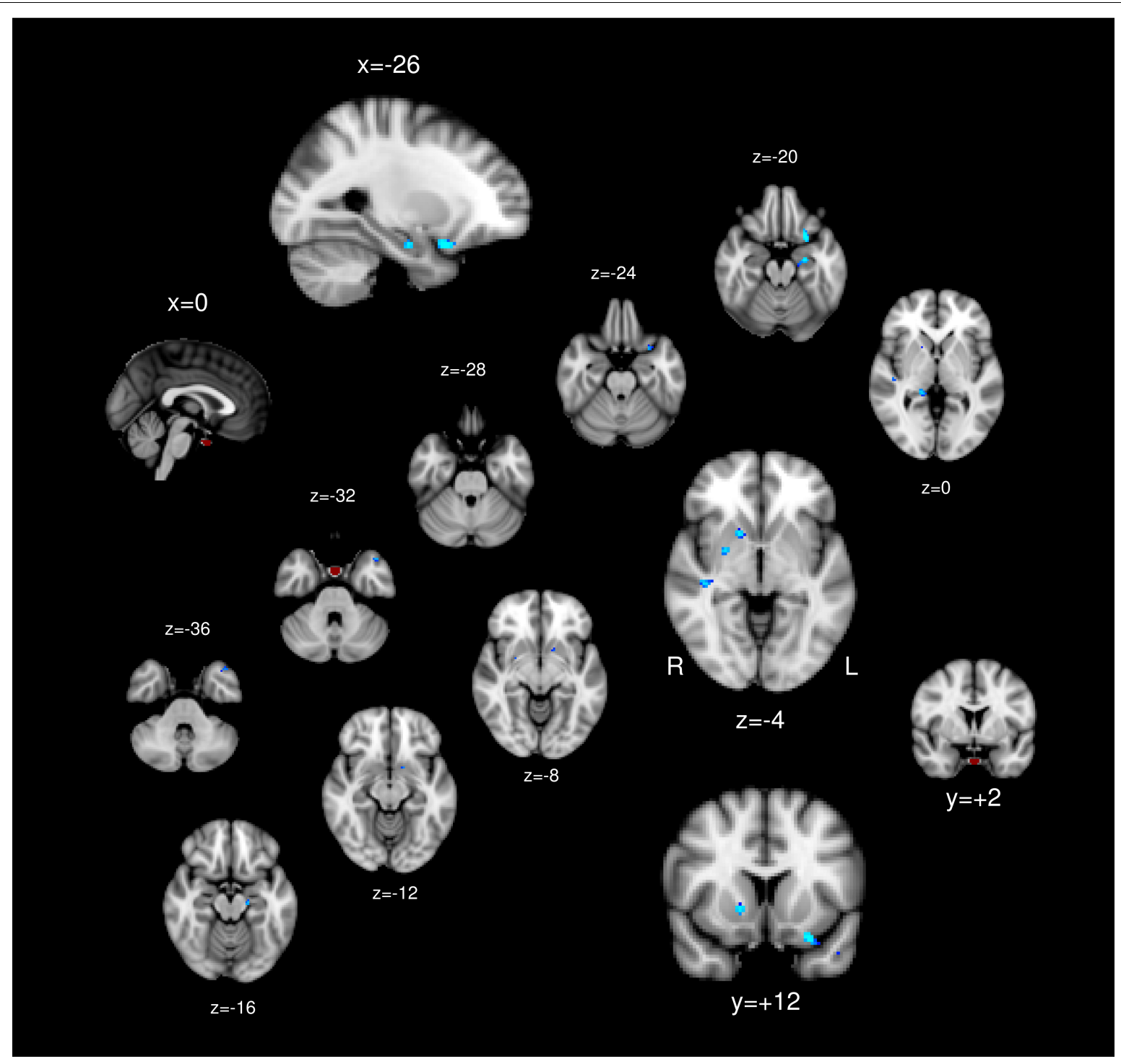

FIGURE 2 | The pituitary $\mathrm{ROI}$ (red) and regions whose pituitary connectivity showed negative association with BMI (blue).

assess the association between the BMI and hypothalamusPG connectivity, a multiple linear regression was calculated to predict the hypothalamus-PG connectivity based on BMI, age, and sex. The regression was not significant, suggesting that the connectivity between hypothalamus-PG connectivity is constant across BMI, while PG and hypothalamus connectivity to the other parts of the brain identified in our voxel-wise analysis are associated with BMI. That is, the PG and hypothalamus are, similarly, influenced by the rest of the brain, resulting in the hypothalamus-PG connectivity remaining constant across BMI. Since the hypothalamus and PG are strongly positively connected, it is expected that the functional connectivity of the hypothalamus would show similar pattern with the PG in its association with BMI.

The orbitofrontal cortex (OFC) showed positive functional connectivity with the PG and inverse association with the BMI in its connectivity to the PG. It is implicated that lesser connectivity between the PG and OFC is associated with BMI. The OFC has been shown to be associated with obesity, as well as being responsible for taste and flavor processing (Small et al., 2007) and food choice (Cohen et al., 2011). Reduced OFC gray matter volume has been found in both adolescent (Ross et al., 2015) and midlife obesity (Driscoll et al., 2011). The OFC volume has been found to predict the risk for obesity (Smucny et al., 2012). More specifically, the OFC has been implicated in its role in preventing overeating. The OFC has been shown to be responsible for disinhibition of eating (Maayan et al., 2012), implicating its importance in preventing obesity. OFC volume has also been found to be associated with reward response in obesity (Shott et al., 2015). Reduced connectivity between the PG and OFC in a more obese population may suggest downregulated control of the OFC over PG. 
The putamen showed inverse association between PG connectivity and BMI. That is, lesser PG-putamen connectivity is implicated in higher BMI. The association between pituitaryputamen dysconnectivity and BMI may be also accounted for by its role in the reward processing since the putamen and PG are both rich in dopamine D2 receptors. Striatal D2 receptors were shown to be reduced in individuals with obesity (Wang et al., 2004) and deficits of D2 receptor availability predicts future weight gain (Michaelides et al., 2012). Antipsychotics, whose pharmacological mechanism is represented by D2 antagonism, is widely known to induce weight gain (Correll et al., 2011). A D2 agonist bromocriptine has been found to counteract obesity (Kok et al., 2006). On the genetic level, polymorphisms in the D2 receptor gene (DRD2) have been implicated in its association to obesity (Nisoli et al., 2007; Ariza et al., 2012), as well as the weight gain response to antipsychotics (Lencz et al., 2010). The D2R profiles in the PG also show association with obesity. Disrupting D2 receptors in the pituitary lactotropes results in weight gain (Perez Millan et al., 2014). Dopaminergic modulations in the PG and striatum are both implicated in their association with BMI.

The PG receives dopaminergic projections from the hypothalamic arcuate nucleus as the terminal of the tuberoinfundibular pathway. Although this may partially account for their lack of BMI-differentiated association, it does not account for the associations with the striatal regions which are independently dopaminergic as the terminal of mesolimbic dopaminergic pathway originating in the substantia niagra. While the striatum and PG are independently dopaminergic in their primary source of their dopaminergic afferents, prolactin has been shown to upregulate both tuberoinfundibular and striatal dopamine neurons. While dopamine is inhibitory against secretion of prolactin (Ben-Jonathan, 1985; Liang et al., 2014), prolactin has been found to promote dopamine discharge in the tuberoinfundibular pathway (Lyons et al., 2012), forming a loop to regulate serum prolactin. In the striatum, rat prolactin increases dopamine turnover in the striatum (Fuxe et al., 1977). Prolonged intake of excessive sucrose has been found to upregulate striatal prolactin, thus showing implication to induce compulsive eating behavior (Ahmed et al., 2014). The prolactinmediated dopaminergic response may be the underlying factor in the association between pituitary-caudate/NAcc functional dysconnectivity and BMI, whereby dopaminergic neurons in the PG and striatum are downregulated by prolactin.

It has to be noted that prolactin would not be the only mechanism that could both interact with the PG and striatum in the complex HPA axis. A voxel-based morphometric study showed a reduced volume of the putamen in obesity and a negative correlation between fasting plasma leptin concentrations and obesity (Pannacciulli et al., 2006), suggesting leptin mediated role of the putamen in regulating food intake. Dysconnectivity between the putamen and PG in obesity could be associated through the mechanism for leptin regulation.

The hippocampus also showed inverse association in its connectivity with the PG, while the hippocampi showed anticorrelation with the PG in the one-sample T-test. It is implicated that the anti-correlation is positively associated with BMI. Whilst the hippocampi also receive dopaminergic afferent from the ventral tegmental area, the association between hippocampus and food intake manipulation as well as physical exercise has been well documented. Physical exercise, which is shown to prevent excessive weight gain, acutely increases hippocampal volume, while hippocampal reduction is found after a series of cafeteria-diet in mice (Sack et al., 2017). Calorie restriction has been shown to improve cognition by upregulating brain-derived neurotrophic factor (BDNF) and downregulating oxidative stress in the hippocampus (Kishi et al., 2015). The hippocampus has been shown to be activated when images of high-calorie food are presented and this activation has been found to be a function of fasting plasma levels of insulin (WallnerLiebmann et al., 2012), suggesting that the hippocampi respond to energy stimuli under the influence of hunger status. Our PGhippocampus dysconnectivity finding may implicate that the PG is regulated by the hippocampus based on the hunger status and food availability.

Several limitations of the current study need to be addressed. First, as this study is based on functional connectivity, where functional connectivity is estimated by co-activations of two regions, causal relationships are not illuminated, although it could be inferred through known endocrinological properties of the PG. Second, despite the known subdivisions within the $\mathrm{PG}$, such as the anterior and posterior pituitary, we avoided to make distinctions due to the imaging resolution of $2 \mathrm{~mm}^{3}$ voxels and smoothing employed in processing. These two regions that showed functional dysconnectivity associated with BMI may arise from two distinct sub-regions of the PG.

It needs to be also addressed that the current study does not make distinction whether the PG connectivity influences BMI or BMI influences PG connectivity. While brain connectivity has been shown to influence body weight specifically in the context of the reward system, body weight status induced by surgical procedures has also been shown to influence functional brain connectivity (Lepping et al., 2015). It remains unclear whether our connectivity findings are the cause or results of the body weight.

In this study, we found functional dysconnectivity between the $P G$ and dopaminergic regions including the putamen, hippocampus, and OFC. The results implicate dopaminergic modulation between the PG and these regions that influences body weight.

\section{ETHICS STATEMENT}

This study was approved by the University of Mississippi Institutional Review Board. This study had no direct involvement of human or animal subjects. All human subjects gave written informed consent.

\section{AUTHOR CONTRIBUTIONS}

PR and TI designed the study and drafted the manuscript. TI analyzed the data. 


\section{FUNDING}

Image preprocessing was performed using the supercomputer cluster at the Mississippi Center for Supercomputing Research partly funded by the National Science Foundation

\section{REFERENCES}

Ahmed, S., Kashem, M. A., Sarker, R., Ahmed, E. U., Hargreaves, G. A., and McGregor, I. S. (2014). Neuroadaptations in the striatal proteome of the rat following prolonged excessive sucrose intake. Neurochem. Res. 39, 815-824. doi: 10.1007/s11064-014-1274-1276

Ariza, M., Garolera, M., Jurado, M. A., Garcia-Garcia, I., Hernan, I., SánchezGarre, C., et al. (2012). Dopamine genes (DRD2/ANKK1-TaqA1 and DRD4-7R) and executive function: their interaction with obesity. PLoS One 7:e41482. doi: 10.1371/journal.pone.0041482

Ben-Jonathan, N. (1985). Dopamine: a prolactin-inhibiting hormone. Endocr. Rev. 6, 564-589. doi: 10.1210/edrv-6-4-564

Biswal, B. B., Mennes, M., Zuo, X.-N., Gohel, S., Kelly, C., Smith, S. M., et al. (2010). Toward discovery science of human brain function. Proc. Natl. Acad. Sci. 107, 4734-4739. doi: 10.1073/pnas.0911855107

Bose, M., Oliván, B., and Laferrère, B. (2009). Stress and obesity: the role of the hypothalamic-pituitary-adrenal axis in metabolic disease. Curr. Opin. Endocrinol. Diabetes Obes. 16, 340-346. doi: 10.1097/MED.0b013e32832fa137

Chalew, S., Nagel, H., and Shore, S. (1995). The hypothalamic-pituitary-adrenal axis in obesity. Obes. Res. 3, 371-382. doi: 10.1002/j.1550-8528.1995.tb00163.x

Clement, K., Vaisse, C., Lahlou, N., Cabrol, S., Pelloux, V., Cassuto, D., et al. (1998). A mutation in the human leptin receptor gene causes obesity and pituitary dysfunction. Nature 392, 398-401. doi: 10.1038/32911

Cohen, J. I., Yates, K. F., Duong, M., and Convit, A. (2011). Obesity, orbitofrontal structure and function are associated with food choice: a cross-sectional study. BMJ Open 1:e000175. doi: 10.1136/bmjopen-2011-2175

Correll, C. U., Lencz, T., and Malhotra, A. K. (2011). Antipsychotic drugs and obesity. Trends Mol. Med. 17, 97-107. doi: 10.1016/j.molmed.2010.10.010

Driscoll, I., Beydoun, M. A., An, Y., Davatzikos, C., Ferrucci, L., Zonderman, A. B., et al. (2011). Midlife obesity and trajectories of brain volume changes in older adults. Hum. Brain Mapp. 33, 2204-2210. doi: 10.1002/hbm.21353

Fox, M. D., and Raichle, M. E. (2007). Spontaneous fluctuations in brain activity observed with functional magnetic resonance imaging. Nat. Rev. Neurosci. 8, $700-711$.

Fuxe, K., Eneroth, P., Gustafsson, J.-A., Löfström, A., and Skett, P. (1977). Dopamine in the nucleus accumbens: preferential increase of DA turnover by rat prolactin. Brain Res. 122, 177-182. doi: 10.1016/0006-8993(77)90677-90671

García-García, I., Jurado, M. Á, Garolera, M., Segura, B., Sala-Llonch, R., MarquésIturria, I., et al. (2013). Alterations of the salience network in obesity: a resting-state fMRI study. Hum. Brain Mapp. 34, 2786-2797. doi: 10.1002/hbm. 22104

Kiem, S. A., Andrade, K. C., Spoormaker, V. I., Holsboer, F., Czisch, M., and Sämann, P. G. (2013). Resting state functional MRI connectivity predicts hypothalamus-pituitary-axis status in healthy males. Psychoneuroendocrinology 38, 1338-1348. doi: 10.1016/j.psyneuen.2012.11.021

Kiparizoska, S., and Ikuta, T. (2017). Disrupted olfactory integration in schizophrenia: functional connectivity study. Int. J. Neuropsychopharmacol. 20, 740-746. doi: 10.1093/ijnp/pyx045

Kishi, T., Hirooka, Y., Nagayama, T., Isegawa, K., Katsuki, M., Takesue, K., et al. (2015). Calorie restriction improves cognitive decline via up-regulation of brain-derived neurotrophic factor. Int. Heart J. 56, 110-115. doi: 10.1536/ihj. 14- 168

Klomp, A., Koolschijn, P. C., Hulshoff Pol, H. E., Kahn, R. S., and Van Haren, N. E. (2012). Hypothalamus and pituitary volume in schizophrenia: a structural MRI study. Int. J. Neuropsychopharmacol. 15, 281-288. doi: 10.1017/ S1461145711000794

Kok, P., Roelfsema, F., Frölich, M., van Pelt, J., Stokkel, M. P. M., Meinders, A. E., et al. (2006). Activation of dopamine D2 receptors simultaneously ameliorates various metabolic features of obese women. Am. J. Physiol. Endocrinol. Metab. 291:E1038. doi: 10.1152/ajpendo.00567.2005
(EPS-0903787). Data used in the preparation of this article were obtained from the Nathan Kline Institute-Rockland Sample (NKI-RS), approved by this respective ethics committee. The NKI-RS project was supported by NIMH BRAINS R01MH094639-01.

Kullmann, S., Heni, M., Veit, R., Ketterer, C., Schick, F., Häring, H.-U., et al. (2012). The obese brain: association of body mass index and insulin sensitivity with resting state network functional connectivity. Hum. Brain Mapp. 33, 1052-1061. doi: $10.1002 / \mathrm{hbm} .21268$

Lencz, T., Robinson, D. G., Napolitano, B., Sevy, S., Kane, J. M., Goldman, D., et al. (2010). DRD2 promoter region variation predicts antipsychotic-induced weight gain in first episode schizophrenia. Pharmacog. Genom. 20:569. doi: 10.1097/FPC.0b013e32833ca24b

Lepping, R. J., Bruce, A. S., Francisco, A., Yeh, H.-W., Martin, L. E., Powell, J. N., et al. (2015). Resting-state brain connectivity after surgical and behavioral weight loss. Obesity 23, 1422-1428. doi: 10.1002/oby.21119

Liang, S.-L., Hsu, S.-C., and Pan, J.-T. (2014). Involvement of dopamine D2 receptor in the diurnal changes of tuberoinfundibular dopaminergic neuron activity and prolactin secretion in female rats. J. Biomed. Sci. 21, 37-37. doi: 10.1186/1423-0127-21-37

Lyons, D. J., Hellysaz, A., and Broberger, C. (2012). Prolactin regulates tuberoinfundibular dopamine neuron discharge pattern: novel feedback control mechanisms in the lactotrophic axis. J. Neurosci. 32:8074. doi: 10.1523/ JNEUROSCI.0129-12.2012

Maayan, L., Hoogendoorn, C., Sweat, V., and Convit, A. (2012). Disinhibited eating in obese adolescents is associated with orbitofrontal volume reductions and executive dysfunction. Obesity 19, 1382-1387. doi: 10.1038/oby.2011.15

Michaelides, M., Thanos, P. K., Kim, R., Cho, J., Ananth, M., Wang, G.-J., et al. (2012). PET imaging predicts future body weight and cocaine preference. NeuroImage 59, 1508-1513. doi: 10.1016/j.neuroimage.2011.08.028

Nakamura, Y., and Ikuta, T. (2017). Caudate-precuneus functional connectivity is associated with obesity preventive eating tendency. Brain Connect. 7, 211-217. doi: 10.1089/brain.2016.0424

Nisoli, E., Nisoli, E., Brunani, A., Borgomainerio, E., and Tonello, C. (2007). D2 dopamine receptor (DRD2) gene Taq1A polymorphism and the eatingrelated psychological traits in eating disorders (anorexia nervosa and bulimia) and obesity. Eat. Weight Disord. 12, 91-96. doi: 10.1007/BF03327583

Nooner, K. B., Colcombe, S. J., Tobe, R. H., Mennes, M., Benedict, M. M., Moreno, A. L., et al. (2012). The NKI-rockland sample: a model for accelerating the pace of discovery science in psychiatry. Front. Neurosci. 6:152. doi: 10.3389/fnins. 2012.00152

Pannacciulli, N., Del Parigi, A., Chen, K., Le, D. S., Reiman, E. M., and Tataranni, P. A. (2006). Brain abnormalities in human obesity: a voxel-based morphometric study. NeuroImage 31, 1419-1425. doi: 10.1016/j.neuroimage. 2006.01.047

Pasquali, R., Vicennati, V., Cacciari, M., and Pagotto, U. (2006). The hypothalamicpituitary-adrenal axis activity in obesity and the metabolic syndrome. Ann. N. Y. Acad. Sci. 1083, 111-128. doi: 10.1196/annals.1367.009

Perez Millan, M. I., Luque, G. M., Ramirez, M. C., Noain, D., Ornstein, A. M., Rubinstein, M., et al. (2014). Selective disruption of dopamine D2 receptors in pituitary lactotropes increases body weight and adiposity in female mice. Endocrinology 155, 829-839. doi: 10.1210/en.2013-1707

Power, J. D., Barnes, K. A., Snyder, A. Z., Schlaggar, B. L., and Petersen, S. E. (2012). Spurious but systematic correlations in functional connectivity MRI networks arise from subject motion. NeuroImage 59, 2142-2154. doi: 10.1016/j. neuroimage.2011.10.018

Power, J. D., Schlaggar, B. L., and Petersen, S. E. (2015). Recent progress and outstanding issues in motion correction in resting state fMRI. NeuroImage 105, 536-551. doi: 10.1016/j.neuroimage.2014.10.044

Ross, N., Yau, P. L., and Convit, A. (2015). Obesity, fitness, and brain integrity in adolescence. Appetite 93, 44-50. doi: 10.1016/j.appet.2015.03.033

Sack, M., Lenz, J. N., Jakovcevski, M., Biedermann, S. V., Falfán-Melgoza, C., Deussing, J., et al. (2017). Early effects of a high-caloric diet and physical exercise on brain volumetry and behavior: a combined MRI and histology study in mice. Brain Imag. Behav. 11, 1385-1396. doi: 10.1007/s11682-016-9638-y 
Shott, M. E., Cornier, M.-A., Mittal, V. A., and Pryor, T. L. (2015). Orbitofrontal cortex volume and brain reward response in obesity. Int. J. Obes. 39, 214-221. doi: 10.1038/ijo.2014.121

Siegel, J. S., Power, J. D., Dubis, J. W., Vogel, A. C., Church, J. A., Schlaggar, B. L., et al. (2014). Statistical improvements in functional magnetic resonance imaging analyses produced by censoring high-motion data points. Hum. Brain Mapp. 35, 1981-1996. doi: 10.1002/hbm.22307

Small, D. M., Bender, G., Veldhuizen, M. G., Rudenga, K., Nachtigal, D., and Felsted, J. (2007). The role of the human orbitofrontal cortex in taste and flavor processing. Ann. N. Y. Acad. Sci. 1121, 136-151. doi: 10.1196/annals.1401.002

Smucny, J., Cornier, M.-A., Eichman, L. C., Thomas, E. A., Bechtell, J. L., and Tregellas, J. R. (2012). Brain structure predicts risk for obesity. Appetite 59, 859-865. doi: 10.1016/j.appet.2012.08.027

Wallner-Liebmann, S., Deisseroth, K., Reishofer, G., Sorantin, E., Blaschitz, B., Kruschitz, R., et al. (2012). Insulin and hippocampus activation in response to images of high-calorie food in normal weight and obese adolescents. Obesity 18, 1552-1557. doi: 10.1038/oby.2010.26

Wang, G.-J., Volkow, N. D., Thanos, P. K., and Fowler, J. S. (2004). Similarity between obesity and drug addiction as assessed by neurofunctional imaging. J. Addict. Dis. 23, 39-53. doi: 10.1300/J069v23n03_04

Wijngaarden, M. A., Veer, I. M., Rombouts, S. A. R. B., van Buchem, M. A., Willems van Dijk, K., Pijl, H., et al. (2015). Obesity is marked by distinct functional connectivity in brain networks involved in food reward and salience. Behav. Brain Res. 287, 127-134. doi: 10.1016/j.bbr.2015. 03.016

Williams, T., Berelowitz, M., Joffe, S. N., Thorner, M. O., Rivier, J., Vale, W., et al. (1984). Impaired growth hormone responses to growth hormonereleasing factor in obesity. N. Engl. J. Med. 311, 1403-1407. doi: 10.1056/ NEJM198411293112203

Conflict of Interest Statement: TI has been a consultant for Sumitomo Dainippon and received speaker's honoraria from Eli Lilly and Daiichi Sankyo, and Dainippon Sumitomo.

The remaining author declares that the research was conducted in the absence of any commercial or financial relationships that could be construed as a potential conflict of interest.

Copyright (c) 2019 Rucker and Ikuta. This is an open-access article distributed under the terms of the Creative Commons Attribution License (CC BY). The use, distribution or reproduction in other forums is permitted, provided the original author(s) and the copyright owner(s) are credited and that the original publication in this journal is cited, in accordance with accepted academic practice. No use, distribution or reproduction is permitted which does not comply with these terms. 